\title{
Craniopharyngioma with intratumoral bleeding coexisting with cerebral venous sinuses thrombosis in a young female; a case report
}

\begin{abstract}
Introduction: Craniopharyngioma is a slow growing intracranial neoplasm, mostly benign in nature and arise from remnants of Rathke's pouch. Incidence is equal among both sexes and can present in all age groups. Acute presentations with headaches are less common especially among adults. Herein we report an adult female with a history of long standing secondary amenorrhea first detected to have a craniopharyngioma when presented acutely with headache and double vision secondary to intrautumoral bleeding.

Case presentation: A 26 year old female with history of secondary amenorrhea and primary hypothyroidism for many years presented with acute onset headache and double vision. On examination she was found to have bilateral papilloedema, left sided lateral rectus palsy and bitemporal hemianopia. She was averagely built and had normal secondary sexual characteristics. A non contrast computerized tomography of the brain revealed a hyperdensity in the pituitary area and magnetic resonance imaging of the brain was suggestive of a craniopharyngioma with bleeding in to tumour. Hormonal assays were in keeping with primary hypothyroidism and hypogonadropic hypogonadism. Since she refused surgery we managed her conservatively with pharmacotherapy including hydrocortisone, thyroxin replacement therapy and gonadotropin treatment for secondary amenorrhea. Review at one month revealed she was free of headache and lateral rectus palsy has resolved but she persisted to have bitemporal hemianopia.
\end{abstract}

Conclusion: In adults craniopharyngiomas could present acutely with headache and double vision due to the rare complication of bleeding in to the tumour.

\section{Case Report}

Volume 7 Issue 2 - 2018

\author{
Kularathna OAR,' Palangasinghe DR,' \\ Pathirana KD, ${ }^{2}$ Herath $\mathrm{HMM}^{3}$ \\ 'University Medical Unit, Teaching Hospital Karapitiya, Galle, Sri \\ Lanka \\ ${ }^{2}$ Consultant Neurologist, Department of Medicine, University of \\ Ruhuna, Sri Lanka \\ ${ }^{3}$ Senior Lecturer in Medicine, University of Ruhuna, Sri Lanka
}

Correspondence: Omalpe Amith Ruwan Kularathna, Registrar in Medicine, University Medical Unit, Teaching Hospital Karapitiya, Galle, Sri Lanka, Tel +94-07785I3896, Email dhammika27@yahoo.com

Received: February 08, 2018 | Published: March 01, 2018

\section{Introduction}

Craniopharyngiomas are rare solid or mixed solid - cystic tumors that arise from remnants of Rathke's pouch. Mostly it is benign in nature ${ }^{1}$ and consists of $3 \%$ of all intracranial neoplasms. ${ }^{2}$ The incidence of craniopharyngioma was 0.13 per 100,000 person's year $^{3}$ and equally common in males and females. There are two histological types - papillary and adamantinomatous. ${ }^{1}$ Adamantinomatous tumour cosists of cysts and dystrophic calcifications ${ }^{5}$ and may develop in all ages. ${ }^{4}$ Papillary type is usually without cyst and calcifications and occurs almost exclusively in children ${ }^{6}$. Peak incidence in adulthood is between 40 to 45 years. Craniopharyngiomas present with hypopituitarism in $95 \%$ of cases, hyperprolactinaemia in $38 \%$, neurological deficits in $67 \%$, opthalmological complaints in $67 \%$ of cases. ${ }^{5}$ Frequently reported acute symptoms were headache, nausea or vomiting and acute visual symptoms, which were more frequent in children than adults. ${ }^{6}$ Craniopharyngiomas with intratumoral bleeding are very rare and in the literature where very few cases were reported. ${ }^{7}$ Here, we present a female patient presented with sub acute onset headache with diplopia and found to have a craniopharyngioma with intratumoral bleeding. She was found to have chronic cerebral venous thrombosis in magnetic resonance venogram (MRV) other than craniopharyngioma.

\section{Case presentation}

A 26 year old unmarried female who was investigated for secondary amenorrhoea and hypothyroidism in the past, presented with sub acute onset headache for one week followed by double vision when looking to lateral side. Headache was mainly confined to frontal region and throbbing in nature without any associated nausea, vomiting. Headache was not worsening with coughing or sneezing. There was no history of fever, seizures or limb weakness. She attained menarche at the age of 12 years and has had infrequent irregular menstrual cycles for one year. Thereafter she became amenorrhic. But she developed normal secondary sexual characteristics. She gained normal height during her puberty. She did not have a proper follow up and not fully evaluated for amenorrhoea. Patient was on oral contraceptive pills intermittently for amenorrhoea with resultant menstrual bleeding. Last three years, patient noticed an excessive weight gain and felt lethargic, excessively sleepy and diagnosed to have hypothyroidism. She was commenced on oral Levothyroxin 50 micrograms/day. She denied history of galactorrhoea, chronic headache, postural dizziness or symptoms suggestive of hypoglycaemia, On physical examination she was conscious and rational. Her body mass index was $25 \mathrm{~kg}$ / $\mathrm{m}^{2}$ and she had normal secondary sexual characteristics. Her blood pressure was $100 / 60 \mathrm{mmHg}$ without any postural hypotension. Neurological examination revealed bilateral papilloedema with left 
sided lateral rectus palsy. Her visual acuity was normal and visual field analysis showed bitemporal hemianopia. Examination of the respiratory system and abdomen revealed no abnormalities.

With the available information on admission we considered the diagnosis of pituitary apoplexy and commenced immediate management including intravenous hydrocortisone and intravenous fluid resuscitation. Her random blood glucose was $114 \mathrm{mg} / \mathrm{dL}$ and serum sodium and potassium levels were $140 \mathrm{mmol} / \mathrm{L}$ and $4.5 \mathrm{mmol} / \mathrm{L}$ respectively. On admission, she was imaged with a non contrast computerized tomography (CT) of brain, which showed a hyper dense lesion in the region of pituitary fossa (Figure 1), without cerebral oedema. It was compatible with our clinical diagnosis and we preceded investigations which revealed following hormone profile. Random Cortisol level was $1120 \mathrm{mmol} / 1$, Serum free thyroxin - 15.6pmol/L $(10-28), \mathrm{TSH}-9 \mathrm{mU} / \mathrm{L}(0.4-4)$, Serum adrenocorticotropic hormone $(\mathrm{ACTH})-26.5 \mathrm{pg} / \mathrm{ml}$ in the morning $(10-60)$, Serum Prolactin - 1308mIU/L, follicular stimulating hormone (FSH) - <0.66mIU/mL (21 - 131), luteinizing hormone (LH)

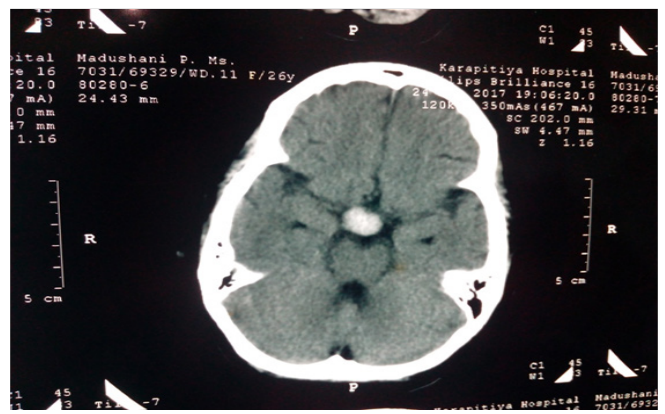

Figure I Non-contrast CT brain showing evidence a hyperdense lesion in the centre in keeping with a bleeding into a tumour.

With the most likely diagnosis of craniopharyngioma with intratumoral bleeding causing bitemporal hemianopia, ophthalmoplegia and hypogonadotrophic hypogonadism we referred the patient for neurosurgical team. While waiting in the neurosurgical ward for surgery over a period of one week she was treated with intravenous hydrocortisone, and she was continued on oral thyroxin $100 \mu \mathrm{g}$ daily. Her headache has gradually improved over a period of one week. Following explanation regarding the need for surgery patient did not consent for operation and wanted a conservative management. She was commenced on gonadotropin therapy in addition to hydrocortisone and thyroxin replacement therapy. Her oral thyroxin dose was increased to $100 \mu \mathrm{g}$ daily. Review after one month of this treatment revealed that she had resolution of headache and lateral rectus palsy. But she had persistent bitemporal hemianopia. It was decided to follow her up monthly in the clinic and a repeat MRI brain was planned to perform in six month to assess the size of the tumour with re-explanation regarding the need for surgery.

\section{Discussion}

This case describes an adult female who had a craniopharyngioma causing hypogonadotrophic hypogonadism, developing intratumoral bleeding presenting acutely with headache, double vision due to left sided lateral rectus and bitemporal hemianopia. Hypothyroidism in our patient was primary in nature and was unrelated to the craniopharyngioma. International literature revealed only few cases of craniopharyngioma with intratumoral bleeding. ${ }^{7}$ Craniopharyngiomas
- $<0.21 \mathrm{mIU} / \mathrm{mL}$ (13 - 88), Serum Oestradiol - 20.9pg/ml (85 -498). Clinical findings, hormonal and biochemical profile were indicating that her pituitary- gonadal axis was affected. She was imaged with magnetic resonance imaging (MRI) brain, which showed supracellar solid tumour with evidences of internal haemmorrhages (Figure 2 )- likely to be a craniopharyngioma $(15 \mathrm{~mm} \times 17 \mathrm{mmx} 12 \mathrm{~mm})$, with optic chiasmal compression and no extension to cavernous sinus or brain stem. Pituitary gland is visualized separately in the pituitary fossa. MRV showed chronic venous thrombosis in superior sagittal, right transverse, and right sigmoid sinuses with multiple draining collateral cerebral veins without evidences of venous infarction. Other laboratory exams were; White cell count $-15400 / \mathrm{mm}^{3}$ - Neutrophils$86 \%$, Lymphocytes $-9 \%$, Haemoglobin $-12.5 \mathrm{~g} / \mathrm{dl}$, Platelet $-200 \mathrm{x}$ $10^{3} / \mathrm{mm}^{3}$, Serum creatinine $-86 \mu \mathrm{mol} / 1$, Serum albumin $-38 \mathrm{~g} / \mathrm{dL}$, Total protein $-55 \mathrm{~g} / \mathrm{dl}$, aspartate transaminase (AST) - $40 \mathrm{IU} / \mathrm{L}$, alanine transaminase (ALT) - $38 \mathrm{IU} / \mathrm{L}$, erythrocyte sedimentation rate (ESR) $-45 \mathrm{~mm}$ in $1^{\text {st }}$ hour, We did not commence her on anticoagulants since there was intratumoral bleeding and the venous sinus thrombosis was chronic without evidence of any infarctions.

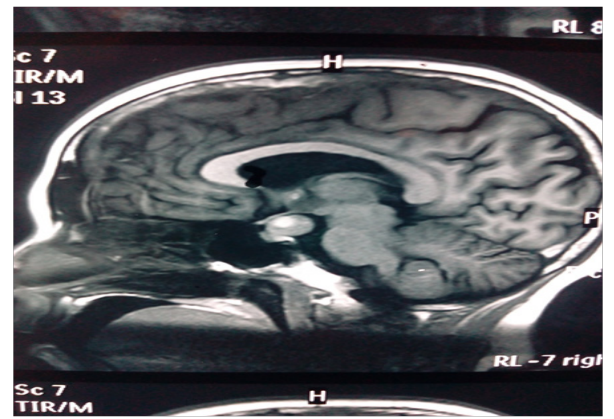

Figure 2 MRI brain demonstrates a suprasellar solid tumour with bleeding into it.

are generally slow growing tumours and symptoms also develop slowly. Therefore delay of diagnosis occurs as in the case of our patient. Menstrual irrigularities or amenorrhoea is common and occurs in $57 \%$ of patients. ${ }^{4}$ Cerebral venous sinus thrombosis is a rare phenomenon and having multifactorial aetiology in our patient. We assumed that reason for the chronic venous sinus thrombosis is usage of oral contraceptive pills in the past. The use of oral contraceptive pills increase the odds of venous thromboembolism by three fold when compared with non users. ${ }^{8}$ Occasionally cerebral venous sinus thrombosis is a presentation of brain tumours. Cases of craniopharyngioma associated with cerebral venous thrombosis are inadequately reported in the literature. Still in our case, this cerebral venous thrombosis may be due to either craniopharyngioma and oral contraceptive pills or an incidental finding. We decided not to treat chronic venous sinus thrombosis with anticoagulation in our patient since there was intratumoral bleeding. Although headache and lateral rectus palsy had gradually improved without surgery according to the patient's wish; careful follow up with repeat neuroimaging and medical treatment is required.

\section{Conclusion}

Although craniopharyngiomas are usually slow growing tumors with indolent course, acute presentations with headache, double vision and ophthalmoplegia are possible even in an adult patient in case of intratumoral bleeding. 


\section{Acknowledgment}

None.

\section{Conflict of interest}

None.

\section{References}

1. Zoia C, Cattalani A, Turpini, et al. Haemorrhagic Presentation of Craniopharyngioma in a Pregnant Woman. Case Rep Neurol Med. 2014;435208.

2. Larijani B, Bastanhagh $\mathrm{MH}$, Pajouhi $\mathrm{M}$, et al. Presentation and outcome of 93 cases of craniopharyngiomas. Eur J Cancer Care (Engl) 2004;13(1):11-15.

3. Bunin GR, Surawicz TS, Witman PA, et al. The descriptive epidemiology of craniopharyngioma. J Neurosurg. 1998;89(4):547-551.
4. Zoicas F, Schöfl C. Craniopharyngioma in Adults. Front Endocrinol (Lausanne). 2012;3:46.

5. Shin JL, Asa SL, Woodhouse LJ, et al. Cystic Lesions of the Pituitary: Clinicopathological Features Distinguishing Craniopharyngioma, Rathke's Cleft Cyst, and Arachnoid Cyst. J Clin Endocrinol Metab. 1999;84(11):3972-3982.

6. Nielsen EH, Jørgensen JO, Bjerre P, et al. Acute presentation of craniopharyngioma in children and adults in a Danish national cohort. Pituitary. 2013;16(4):528-535.

7. Yamashita S, Matsumoto Y, Kunishio K, et al. Craniopharyngiomas with Intratumoral Hemorrhage. Neurol Med Chir (Tokyo). 2004;44(1):43-46.

8. Baratloo A, Safari S, Rouhipour A, et al. The Risk of Venous Thromboembolism with Different Generation of Oral Contraceptives; a Systematic Review and Meta-Analysis. Emerg (Tehran). 2014;2(1):1-11. 